\title{
AN INVERSE SPECTRAL PROBLEM FOR STURM-LIOUVILLE OPERATORS WITH DISCONTINUOUS COEFFICIENTS
}

\author{
ROBERT CARLSON \\ (Communicated by Hal L. Smith)
}

\begin{abstract}
For generic Sturm-Liouville problems with piecewise constant leading coefficients, the leading coefficient can be determined up to a finite ambiguity from the eigenvalues of the problem.
\end{abstract}

\section{INTRODUCTION}

Ordinary differential operators with discontinuous leading coefficients occasionally arise in scientific modelling; for instance [11] and [8] discuss such a geophysical application, while [10] gives examples in electromagnetics and elasticity. This paper addresses the inverse spectral problem of recovering information about the coefficients of Sturm-Liouville operators from the sequence of eigenvalues. The case we analyze is the case when the leading coefficient has a finite number of jump discontinuities. Our aim is to determine, to the extent possible, the location and magnitude of these jumps from the spectrum of a single Sturm-Liouville problem.

Inverse eigenvalue problems, usually for equations of the form

$$
-y^{\prime \prime}+q(x) y=\lambda y
$$

with a variety of boundary conditions, have an extensive literature. Unless $q(x)$ is constrained, one needs the spectra from two sets of boundary conditions, or one spectrum and a sequence of norming constants, to uniquely determine $q(x)$. Early important work on these problems was done by Borg [3], followed shortly thereafter by extensive work in the Soviet Union [6,9]. More recently, new geometric ideas were introduced by McKean and Trubowitz and their coworkers (see [15] and the references therein). There have been several works addressing inverse eigenvalue problems with less-regular coefficients. Most of these are motivated by geophysical models for oscillations of the earth. Hald [8] examines problems with an interior jump condition. Andersson [1] and Coleman and McLaughlin [5] consider problems of the form

$$
\left(p^{2} y^{\prime}\right)^{\prime}+\lambda p^{2} y=0
$$

Received by the editors May 14, 1992.

1991 Mathematics Subject Classification. Primary 34A55. 
with coefficients too irregular for the classical reduction to Liouville normal form. For surveys of these and related results and extensive lists of references one can consult [12] and [4].

The problem we consider is

$$
\begin{gathered}
-\left[1 / p(x)^{2}\right] \varphi^{\prime \prime}+q(x) \varphi=\lambda \varphi, \\
a \varphi^{\prime}(0)-b \varphi(0)=0, \quad c \varphi^{\prime}(1)-d \varphi(1)=0
\end{gathered}
$$

with $a^{2}+b^{2}$ and $c^{2}+d^{2}$ positive, where $q(x) \in L^{1}[0,1]$ and $p(x)$ is a piecewise constant positive function with value $p_{n}$ on the intervals $\left(x_{n}, x_{n+1}\right)$, $n=0, \ldots, N-1$, and with $0=x_{0}<x_{1}<\cdots<x_{N}=1$. We require that $\varphi(x)$ and $\varphi^{\prime}(x)$ are continuous on $[0,1]$ and satisfy the differential equation on the open intervals $\left(x_{n}, x_{n+1}\right)$.

Elementary examples show that in general $p$ cannot be determined from the eigenvalues of the problem (1.a). If $q(x)=0$ and $a=c=0$, then the two problems with coefficients $p_{1}(x)$ and $p_{2}(x)$ will have the same eigenvalues if $p_{1}(x)=p_{2}(1-x)$. A more interesting family of examples on the interval $[0, \pi]$ was pointed out in [7]. If $0<\alpha<1$, it is easy to show that the following problems all have the same eigenvalues:

$$
-\left[1 / p(x)^{2}\right] y^{\prime \prime}=\lambda y, \quad y(0)=0=y(\pi)
$$

where $p(x)=1 /(2 \alpha)$ for $0<x<\alpha \pi$ and $p(x)=1 /(2(1-\alpha))$ for $\alpha \pi<x<\pi$.

To state the main result it will be convenient to introduce some notation. Let $l_{n}=x_{n+1}-x_{n}$ and $\zeta_{n}=l_{n} p_{n}, n=0, \ldots, N-1$. Let $\mu_{n}=p_{n} / p_{n+1}$ for $n=0, \ldots, N-2$. Denote by $B_{i}=(\beta(0, i), \ldots, \beta(N-1, i))$ an $N$-tuple whose entries have values \pm 1 . One of our hypotheses will be that the numbers $\sum_{m=0}^{N-1} \beta(m, i) \zeta_{m}$ are distinct if the vectors $B_{i}$ are distinct. Notice that for distinct $B_{i}$ two such sums agree when $\left(\zeta_{0}, \ldots, \zeta_{N-1}\right)$ lies in a hyperplane, so asking for the sums to be distinct is the condition that $\left(\zeta_{0}, \ldots, \zeta_{N-1}\right)$ lie outside a finite number of hyperplanes.

Thecrem 1.1. Suppose $\left(\zeta_{0}, \ldots, \zeta_{N-1}\right)$ is such that the sums $\sum_{m=0}^{N-1} \beta(m, i) \zeta_{m}$ are distinct if $(\beta(0, i), \ldots, \beta(N-1, i))$ are distinct vectors with entries \pm 1 . Suppose that $\mu_{n} \neq 1$ for $n=0, \ldots, N-2$. If two problems (1.a) have the same eigenvalues, then the interval lengths and corresponding constants $\left(l_{n}, p_{n}\right)$, $n=0, \ldots, N-1$, are the same up to a permutation of $0, \ldots, N-1$.

In outline form, the proof of Theorem 1.1 is straightforward. As in the case with $p=1$ the eigenvalues of problem (1.a) are the zeros of an entire function $F_{q}(\lambda)$, which has order $\frac{1}{2}$ and only simple zeros (Lemma 3.1); by Hadamard's theorem [16, p. 74] $F_{q}(\lambda)$ is determined up to a constant multiple by its zeros. Problem (1.a) can be treated as a perturbation of the case when $q=0$. For the case $q=0$ it is possible to find an explicit formula for $F_{0}(\lambda)$. In fact, it has the form

$$
\begin{aligned}
F_{0}(\lambda)=\sum_{i \in I} e_{i}\left\{\beta(N-1, i) a c \mu_{N-1} \omega \sin \left(f_{i} \omega\right)\right. \\
\left.+\left[a d+\beta(N-1, i) b c \mu_{N-1} / p_{0}\right] \cos \left(f_{i} \omega\right)+b d \sin \left(f_{i} \omega\right) /\left[p_{0} \omega\right]\right\}
\end{aligned}
$$

where $f_{i}=\sum_{m=0}^{N-1} \beta(m, i) \zeta_{m}$ and $\omega=\lambda^{1 / 2}$. The frequencies $f_{i}$ and the 
coefficients can be determined by considering expressions like

$$
\lim _{L \rightarrow \infty}[2 / L] \int_{1}^{L} F_{0}(\lambda) \sin (\nu \omega) / \omega d \omega
$$

for $\nu>0$. Some elementary algebra then gives the result.

By a change of variables it is possible to show that more general boundary value problems will have the same eigenvalues as one of the problems (1.a). Consider first a problem of the form

$$
\begin{gathered}
-R(x)\left[P_{1}(x) u^{\prime}\right]^{\prime}+Q_{1}(x) u=\lambda u, \\
k_{1} u^{\prime}(0)+k_{2} u(0)=0, \quad k_{3} u^{\prime}(1)+k_{4} u(1)=0 .
\end{gathered}
$$

It is assumed that $R(x), 1 / R(x), P_{1}(x)$, and $1 / P_{1}(x)$ are positive and bounded and that $P_{1}(x)$ is continuous except for a finite number of jump discontinuities. It is pointed out in [13] that the change of variables $t=\left[\int^{x} P^{-1}(s)\right] /$ $\left[\int_{0}^{1} P^{-1}(s) d s\right]$ and $\varphi(t)=u(x(t))$ leads to an equation $-P_{2}(x) \varphi^{\prime \prime}+Q_{2}(x) \varphi=$ $\lambda \varphi$ with a simple change in end point boundary conditions and the location of the discontinuities. Also the jump conditions for $u$ natural at the discontinuities of problem (1.c) become simply the requirement that $\varphi$ be continuous with continuous first derivative everywhere. To further simplify the problem, note that if $P_{2}(x)$ is continuous except for finitely many jump discontinui:ies, then $P_{2}(x)=p(x) J(x)$ where $J(x)$ is continuous while $p(x)$ is piecewise constant with jumps at the same locations as $P_{2}(x)$. Finally a standard change of variables [2, p. 296] reduces the problem to the form (1.a).

\section{THE CASE $q(x)=0$}

The analysis of problem (1.a) will begin with the case $q(x)=0$. Let $\omega=$ $\lambda^{1 / 2}$ with $\omega>0$ when $\lambda>0$. On the interval $\left(x_{n}, x_{n+1}\right)$ any solution of $-y^{\prime \prime}=\lambda p(x)^{2} y$ must have the form

$$
y(x)=A_{n} \cos \left(p_{n} \omega\left[x-x_{n}\right]\right)+\left(B_{n} / \omega\right) \sin \left(p_{n} \omega\left[x-x_{n}\right]\right) .
$$

There is a linear map from the coefficients

$$
\left[\begin{array}{l}
A_{n} \\
B_{n}
\end{array}\right] \text { to }\left[\begin{array}{l}
A_{n+1} \\
B_{n+1}
\end{array}\right]
$$

which can be computed using the continuity of $y$ and $y^{\prime}$. The matrix taking the basis elements $\cos \left(p_{n} \omega\left[x-x_{n}\right]\right)$ and $\sin \left(p_{n} \omega\left[x-x_{n}\right]\right) / \omega$ to a linear combination of $\cos \left(p_{n+1} \omega\left[x-x_{n+1}\right]\right)$ and $\sin \left(p_{n+1} \omega\left[x-x_{n+1}\right]\right) / \omega$ is

where

$$
T_{n}=\left[\begin{array}{ll}
a_{n} & b_{n} \\
c_{n} & d_{n}
\end{array}\right]
$$

$$
\begin{array}{ll}
a_{n}=\cos \left(p_{n} \omega l_{n}\right), & b_{n}=\sin \left(p_{n} \omega l_{n}\right) / \omega, \\
c_{n}=-\omega p_{n} \sin \left(p_{n} \omega l_{n}\right) / p_{n+1}, & d_{n}=p_{n} \cos \left(p_{n} \omega l_{n}\right) / p_{n+1} .
\end{array}
$$

Notice that the entries of $T_{n}$ are entire functions of $\lambda$ and that

$$
T_{n}=\left[\begin{array}{cc}
1 & 0 \\
0 & \omega
\end{array}\right]\left[\begin{array}{cc}
\cos \left(p_{n} l_{n} \omega\right) & \sin \left(p_{n} l_{n} \omega\right) \\
-p_{n} \sin \left(p_{n} l_{n} \omega\right) / p_{n+1} & p_{n} \cos \left(p_{n} l_{n} \omega\right) / p_{n+1}
\end{array}\right]\left[\begin{array}{cc}
1 & 0 \\
0 & 1 / \omega
\end{array}\right] .
$$

Let $G(\omega)=\left[\begin{array}{ll}1 & 0 \\ 0 & \omega\end{array}\right]$ and $G^{-1}(\omega)=\left[\begin{array}{cc}1 & 0 \\ 0 & 1 / \omega\end{array}\right]$.

The following lemma is the result of direct computation. 


\section{Lemma 2.1 .}

$$
\begin{gathered}
{\left[\begin{array}{cc}
\cos (b) & \sin (b) \\
-\mu_{b} \sin (b) & \mu_{b} \cos (b)
\end{array}\right]\left[\begin{array}{cc}
\cos (a) & \sin (a) \\
\mp \mu_{a} \sin (a) & \pm \mu_{a} \cos (a)
\end{array}\right]} \\
\quad=\frac{\left(1 \pm \mu_{a}\right)}{2}\left[\begin{array}{cc}
\cos (a+b) & \sin (a+b) \\
-\mu_{b} \sin (a+b) & \mu_{b} \cos (a+b)
\end{array}\right] \\
\quad+\frac{\left(1 \mp \mu_{a}\right)}{2}\left[\begin{array}{cc}
\cos (a-b) & \sin (a-b) \\
\mu_{b} \sin (a-b) & -\mu_{b} \cos (a-b)
\end{array}\right]
\end{gathered}
$$

Let

$$
M(t)=\left[\begin{array}{cc}
\cos (t) & \sin (t) \\
-\sin (t) & \cos (t)
\end{array}\right]
$$

The next lemma provides a representation for the composition $T_{n} \cdots T_{0}$.

Lemma 2.2. For $n>0, T_{n} \cdots T_{0}=G(\omega)\left[\sum_{i \in I_{n}} C_{i}\right] G^{-1}(\omega)$ where the index set $I_{n}$ is the set of distinct vectors $B_{i}=(\beta(0, i), \beta(1, i), \ldots, \beta(n, i))$ with $\beta(0, i)=1, \beta(m, i)= \pm 1$ for $m=1, \ldots, n$ and the matrices $C_{i}$ are given by

$$
\begin{aligned}
C_{i}= & 2^{-n}\left[\prod_{m=0}^{n-1}\left(1+\beta(m, i) \beta(m+1, i) \mu_{m}\right)\right] \\
& \times\left[\begin{array}{cc}
1 & 0 \\
0 & \beta(n, i) \mu_{n}
\end{array}\right] M\left(\omega\left[\sum_{m=0}^{n} \beta(m, i) \zeta_{m}\right]\right) .
\end{aligned}
$$

Proof. The proof is by induction, using Lemma 2.1 as the essential tool. The initial case $n=1$ is easily checked. Let $S_{n}=G^{-1}(\omega) T_{n} G(\omega)$. When we multiply $T_{n-1} \cdots T_{0}$ on the left by $T_{n}$, we find

$$
T_{n} T_{n-1} \cdots T_{0}=G(\omega)\left[\sum_{i \in I_{n-1}} S_{n} C_{i}\right] G^{-1}(\omega),
$$

with $I_{n-1}$ running over the distinct vectors $(\beta(0, i), \ldots, \beta(n-1, i))$. Putting aside for the moment the scalar factor $2^{-n+1} \prod_{m=0}^{n-2}(1+\beta(m, i) \beta(m+1, i))$, we examine the product

$$
S_{n}\left[\begin{array}{cc}
1 & 0 \\
0 & \beta(n-1, i) \mu_{n-1}
\end{array}\right] M\left(\omega\left[\sum_{m=0}^{n-1} \beta(m, i) \zeta_{m}\right]\right),
$$

which by Lemma 2.1 is equal to

$$
\begin{aligned}
& {\left[\left(1+\beta(n-1, i) \mu_{n-1}\right) / 2\right]\left[\begin{array}{cc}
1 & 0 \\
0 & \mu_{n}
\end{array}\right] M\left(\omega\left[\zeta_{n}+\sum_{m=0}^{n-1} \beta(m, i) \zeta_{m}\right]\right)} \\
& \quad+\left[\left(1-\beta(n-1, i) \mu_{n-1}\right) / 2\right]\left[\begin{array}{cc}
1 & 0 \\
0 & -\mu_{n}
\end{array}\right] M\left(\omega\left[-\zeta_{n}+\sum_{m=0}^{n-1} \beta(m, i) \zeta_{m}\right]\right) .
\end{aligned}
$$

Examining (2.a) we see that the power of 2 is correct. Now each of the vectors $(\beta(0, i), \ldots, \beta(n, i))$ appearing in (2.a) is just one of the vectors $(\beta(0, i), \ldots$, $\beta(n-1, i))$ augmented in the last place with \pm 1 . It is exactly this augmentation that we see in (2.b), establishing the lemma. 
Next the eigenvalues of the $q(x)=0$ problem will be expressed as the zeros of an elementary function. Using the boundary conditions $a y^{\prime}(0)-b y(0)=0$ and $c y^{\prime}(0)-d y(1)=0$, we see that for all $\lambda$ any eigenfunction has the form

$$
\left[\begin{array}{c}
a \\
b / p_{0}
\end{array}\right]
$$

in the basis $\cos \left(p_{0} \omega x\right), \sin \left(p_{0} \omega x\right) / \omega$ which is valid in the first interval. On the last interval the eigenfunction is represented by the vector

$$
T_{N-2} \cdots T_{0}\left[\begin{array}{c}
a \\
b / p_{0}
\end{array}\right]
$$

Writing $T_{N-2} \cdots T_{0}$ as

we get our eigenfunction

$$
\tau(\lambda)=\left[\begin{array}{ll}
\tau_{11} & \tau_{12} \\
\tau_{21} & \tau_{22}
\end{array}\right]
$$

$$
\begin{aligned}
& {\left[\tau_{11} a+\tau_{12} b / p_{0}\right] \cos \left(p_{N-1} \omega\left[x-x_{N-1}\right]\right)} \\
& \quad+\left[\tau_{21} a+\tau_{22} b / p_{0}\right] \sin \left(p_{N-1} \omega\left[x-x_{N-1}\right]\right) / \omega
\end{aligned}
$$

and the equation defining the eigenvalues is $F_{0}(\lambda)=0$ where

$$
\begin{aligned}
F_{0}(\lambda)= & \left.d\left[\tau_{11} a+\tau_{12} b / p_{0}\right] \cos \left(p_{N-1} \omega l_{N-1}\right]\right) \\
& \left.+d\left[\tau_{21} a+\tau_{22} b / p_{0}\right] \sin \left(p_{N-1} \omega l_{N-1}\right]\right) / \omega \\
& \left.+c\left[\tau_{11} a+\tau_{12} b / p_{0}\right] p_{N-1} \omega \sin \left(p_{N-1} \omega l_{N-1}\right]\right) \\
& \left.-c\left[\tau_{21} a+\tau_{22} b / p_{0}\right] p_{N-1} \cos \left(p_{N-1} \omega l_{N-1}\right]\right)
\end{aligned}
$$

If we define $p_{N}=1$ and $\mu_{N-1}=p_{N-1}$ then it is a simple computation to verify that

$$
F_{0}(\lambda)=[d,-c] T_{N-1} \cdots T_{0}\left[\begin{array}{c}
a \\
b / p_{0}
\end{array}\right] .
$$

It is also elementary that $F_{0}(\lambda)$ is an entire function of order $1 / 2$.

It will also be helpful to have a representation for the eigenfunctions $y_{1}(x, \lambda)$, $y_{2}(x, \lambda)$ which satisfy the equation $-\left(1 / p^{2}\right) D^{2} y=\lambda y$ and the usual initial conditions $y_{j}^{(i-1)}(0, \lambda)=\delta_{i j}, i, j=1,2$. For any $x \in[0,1], x$ will lie in an interval $\left[x_{n}, x_{n+1}\right]$. In this interval the eigenfunctions will have the form

$$
\begin{aligned}
& y_{1}(x, \lambda)=\left[\cos \left(p_{n} \omega\left[x-x_{n}\right]\right), \sin \left(p_{n} \omega\left[x-x_{n}\right]\right) / \omega\right] T_{n-1} \cdots T_{0}\left[\begin{array}{l}
1 \\
0
\end{array}\right], \\
& y_{2}(x, \lambda)=\left[\cos \left(p_{n} \omega\left[x-x_{n}\right]\right), \sin \left(p_{n} \omega\left[x-x_{n}\right]\right) / \omega\right] T_{n-1} \cdots T_{0}\left[\begin{array}{c}
0 \\
1 / p_{0}
\end{array}\right] .
\end{aligned}
$$

Using the expression for $T_{n-1} \cdots T_{0}$ from Lemma 2.2 it is easy to compute that

$$
\begin{aligned}
y_{1}(x, \lambda)=\sum & {\left[c_{k} \cos \left(\omega f_{k}\right) \cos \left(p_{n} \omega\left[x-x_{n}\right]\right)\right.} \\
& \left.+C_{k} \omega \sin \left(\omega f_{k}\right) \sin \left(p_{n} \omega\left[x-x_{n}\right]\right) / \omega\right], \\
y_{2}(x, \lambda)=\sum[ & d_{k} \cos \left(\omega f_{k}\right) \sin \left(p_{n} \omega\left[x-x_{n}\right]\right) / \omega \\
& \left.+D_{k} \sin \left(\omega f_{k}\right) \cos \left(p_{n} \omega\left[x-x_{n}\right]\right) / \omega\right]
\end{aligned}
$$

where the terms $c_{k}, C_{k}, d_{k}, D_{k}$, and $f_{k}$ depend on the interval $\left[x_{n}, x_{n+1}\right]$ and on the values $\mu_{m}$ and $\zeta_{m}$ but not on $\lambda$. 
Theorem 1.1 will now be proven in the special case $q(x)=0$. Writing

$$
F_{0}(\lambda)=[d,-c] G(\omega)\left[\sum_{i \in I_{N-1}} C_{i}\right] G^{-1}(\omega)\left[\begin{array}{c}
a \\
b / p_{0}
\end{array}\right]
$$

and letting $e_{i}=2^{-N+1} \prod_{m=0}^{N-2}\left(1+\beta(m, i) \beta(m+1, i) \mu_{m}\right)$ for $i \in I_{N-1}$ we find that

$$
\begin{array}{r}
F_{0}(\lambda)=\sum_{i \in I_{N-1}} e_{i}\left\{\beta(N-1, i) a c \mu_{N-1} \omega \sin \left(f_{i} \omega\right)\right. \\
+\left[a d+\beta(N-1, i) b c \mu_{N-1} / p_{0}\right] \cos \left(f_{i} \omega\right) \\
\left.+b d \sin \left(f_{i} \omega\right) /\left[p_{0} \omega\right]\right\}
\end{array}
$$

where $f_{i}=\sum_{m=0}^{N-1} \beta(m, i) \zeta_{m}$. Notice, in particular, that $e_{i} \neq 0$ for all $i \in$ $I_{N-1}$ and that if $a^{2}+b^{2}>0$ and $c^{2}+d^{2}>0$ then at least one of $a c \mu_{N-1}$, $\left[a d+\beta(N-1, i) b c \mu_{N-1} / p_{0}\right]$, or $b d / p_{0}$ is not zero. In short, all the frequencies which appear to be present are in fact there.

There is an algorithm for recovering, up to a permutation, the function $p(x)$ from the eigenvalues of problem (1.a). Consider

$$
\lim _{L \rightarrow \infty}[2 / L] \int_{1}^{L} F_{0}(\lambda) \omega^{-1} \sin (\nu \omega) d \omega
$$

for $\nu$ positive. These limits will all be zero if and only if $a c=0$. If $a c \neq 0$, the frequencies $f_{i}$ are determined, and the coefficients $e_{i}$ are determined up to a single scalar multiple. If the limits in (2.d) are all zero then this data is recovered from

$$
\lim _{L \rightarrow \infty}[2 / L] \int_{1}^{L} F_{0}(\lambda) \cos (\nu \omega) d \omega
$$

or from

$$
\lim _{L \rightarrow \infty}[2 / L] \int_{1}^{L} F_{0}(\lambda) \omega \sin (\nu \omega) d \omega .
$$

Because of the assumption that the frequencies $f_{i}$ are distinct, it is easy to determine the numbers $\zeta_{m}$, up to a permutation, from the $f_{i}$. For convenience of notation assume that $\zeta_{0}<\cdots<\zeta_{N-1}$. Let $S=\sum \zeta_{m}$ and $S_{k}=\left[\sum \zeta_{m}\right]-$ $2 \zeta_{k}$. Since the $\zeta_{m}$ are all positive, the largest two observed positive frequencies are $S$ and $S_{0}$; their difference is $2 \zeta_{0}$. Proceeding inductively, suppose we have identified $\zeta_{0}, \ldots, \zeta_{k}$. Discard all observed positive frequencies of the form $\sum_{m=k+1}^{N-1} \zeta_{m}+\sum_{m=0}^{k}( \pm) \zeta_{m}$. The largest remaining frequency must be $S_{k+1}$, which gives us $\zeta_{k+1}$. Of course, this procedure only determines $\zeta_{0}, \ldots, \zeta_{N-1}$ up to a permutation since their size order is unknown.

The remaining steps will be carried out for each permutation of $\zeta_{0}, \ldots, \zeta_{N-1}$. With each hypothesized permutation we can generate the distinct frequencies $\sum \beta(m, i) \zeta_{m}$ and find the (hypothesized) coefficients $e_{i}, i \in I_{N-1}$. For each $m, 0 \leq m \leq N-1$, examine the two coefficients $\varepsilon_{k}$ obtained when $i$ is selected so that $\beta(0,1)=\cdots=\beta(k, i)=1, \beta(k+1, i)=\cdots=\beta(N-1, i)=$ -1 , and $e$ obtained when $\beta(0, i)=\cdots=\beta(N-1, i)=1$. Notice that $\varepsilon_{k}$ will have factors $\left(1+\mu_{m}\right)$ except for the single factor $\left(1-\mu_{k}\right)$ and that the corresponding factors of $e$ will all be $\left(1+\mu_{m}\right)$. Defining $E_{k}=\varepsilon_{k} / e$, we find $\left[1-\mu_{k}\right] /\left[1+\mu_{k}\right]=E_{k}$ or $\mu_{k}=\left[1-E_{k}\right] /\left[1+E_{k}\right]$. Thus for each of our candidate 
permutations we can identify $\mu_{0}, \ldots, \mu_{N-2}$. (Note that the unknown scalar dropped out.)

So far we have determined $\zeta_{0}, \ldots, \zeta_{N-1}$ up to permutation and, for each permutation, the hypothesized values of $\mu_{0}, \ldots, \mu_{N-2}$. To finish note that the total length of the interval is $1=\sum_{m=0}^{N-1} l_{m}$. We have $p_{m}=p_{0} /\left[\mu_{0} \cdots \mu_{m-1}\right]$ and $\zeta_{m}=p_{m} l_{m}$, so that $1=\sum l_{m}=\sum \zeta_{m} / p_{m}=\left[1 / p_{0}\right] \sum_{m=0}^{N-1} \zeta_{m}\left\{\prod_{j=0}^{m-1} \mu_{j}\right\}$ gives us $p_{0}$. Finally, for each hypothesized permutation the corresponding operator (with $q(x)=0$ ) can be constructed, and those giving the correct set of eigenvalues (or $F(\lambda))$ determined.

\section{THE GENERAL CASE}

Let $\varphi(x, \lambda)$ be the solution of the initial value problem

$$
-\left[1 / p^{2}(x)\right] \varphi^{\prime \prime}+q(x) \varphi=\lambda \varphi, \quad \varphi(0, \lambda)=a, \quad \varphi^{\prime}(0, \lambda)=b,
$$

and let $F_{q}(\lambda)=d \varphi(1, \lambda)-c \varphi^{\prime}(1, \lambda) . F_{q}(1, \lambda)$ is an entire function whose zeros are the eigenvalues of problem (1.a). Define $z(x, \lambda)=\partial \varphi(x, \lambda) / \partial \lambda$, and note that $z$ satisfies the initial value problem

$$
-\left[1 / p^{2}(x)\right] z^{\prime \prime}+q(x) z=\varphi+\lambda z, \quad z(0, \lambda)=0, \quad z^{\prime}(0, \lambda)=0 .
$$

Following part of the proof of [15, Theorem 2.2] we have

Lemma 3.1. $F_{q}(\lambda)$ has only simple zeros.

Proof. Multiplying the equation in (3.a) by $z$ and the equation in (3.b) by $\varphi$ and subtracting we find that

$$
\varphi^{\prime \prime} z-\varphi z^{\prime \prime}=\left[\varphi^{\prime} z-\varphi z^{\prime}\right]^{\prime}=p^{2} \varphi^{2} .
$$

Integration gives

$$
\varphi^{\prime}(1, \lambda) z(1, \lambda)-\varphi(1, \lambda) z^{\prime}(1, \lambda)=\int_{0}^{1} p^{2}(x) \varphi^{2}(x, \lambda) d x>0 .
$$

Thus the two equations $F_{q}(\lambda)=c \varphi(1, \lambda)+d \varphi^{\prime}(1, \lambda)=0$ and $\partial F_{q}(\lambda) / \partial \lambda=$ $c z(1, \lambda)+d z^{\prime}(1, \lambda)=0$ cannot be satisfied simultaneously.

The proof of Theorem 1.1 will be completed by using standard techniques to estimate the growth of solutions of the initial value problems

$$
\begin{gathered}
-\left[1 / p^{2}(x)\right] D^{2} \varphi_{j}(x)+q(x) \varphi_{j}(x)=\lambda \varphi_{j}(x), \\
\varphi_{j}^{(i-1)}(0, \lambda)=\delta_{i j}, \quad i, j,=1,2 .
\end{gathered}
$$

The estimates are based on the solutions $y_{1}(x, \lambda), y_{2}(x, \lambda)$ previously developed for the case $q(x)=0$. We begin with the variation of parameters representations

$$
\begin{aligned}
\varphi_{j}(x, \lambda)= & y_{j}(x, \lambda) \\
& +\int_{0}^{x}\left[y_{1}(t, \lambda) y_{2}(x, \lambda)-y_{1}(x, \lambda) y_{2}(t, \lambda)\right] p^{2}(t) q(t) \varphi_{j}(t, \lambda) d t, \\
\varphi_{j}^{\prime}(x, \lambda)= & y_{j}^{\prime}(x, \lambda) \\
& +\int_{0}^{x}\left[y_{1}(t, \lambda) y_{2}^{\prime}(x, \lambda)-y_{1}^{\prime}(x, \lambda) y_{2}(t, \lambda)\right] p^{2}(t) q(t) \varphi_{j}(t, \lambda) d t .
\end{aligned}
$$


Let $\Phi(x, t, \lambda)=y_{1}(t, \lambda) y_{2}(x, \lambda)-y_{1}(x, \lambda) y_{2}(t, \lambda)$. Observe that for each $t$ the function $\Phi(x, t, \lambda)$ satisfies the equation $-\Phi^{\prime \prime}(x, t, \lambda)=\lambda p^{2}(x) \Phi(x, t, \lambda)$ with the initial conditions $\Phi(t, t, \lambda)=0$ and $(d / d x) \Phi(t, t, \lambda)=y_{1}(t, \lambda) y_{2}^{\prime}(t, \lambda)$ $-y_{1}^{\prime}(t, \lambda) y_{2}(t, \lambda)=1$ since it is just the Wronskian of the two solutions $y_{1}, y_{2}$.

Now if $x_{n} \leq t<x \leq x_{n+1}$ then $\Phi(x, t, \lambda)=\sin \left(p_{n} \omega[x-t]\right) /\left[p_{n} \omega\right]$. On the other hand, if $x_{n} \leq t<x_{n+1}<x$ then the solution crosses one of the jumps, and after applying the appropriate transition matrix we find that

$$
\begin{aligned}
\Phi(x, t, \lambda)= & \sin \left(p_{n} \omega\left[x_{n+1}-t\right]\right) \cos \left(p_{n+1} \omega\left[x-x_{n+1}\right]\right) /\left[p_{n} \omega\right] \\
& +\cos \left(p_{n} \omega\left[x_{n+1}-t\right]\right) \sin \left(p_{n+1} \omega\left[x-x_{n+1}\right]\right) /\left[p_{n+1} \omega\right] \\
= & {[1 / 2]\left[1 / p_{n}+1 / p_{n+1}\right] \sin \left(p_{n} \omega\left[x_{n+1}-t\right]+p_{n+1} \omega\left[x-x_{n+1}\right]\right) / \omega } \\
& +[1 / 2]\left[1 / p_{n}-1 / p_{n+1}\right] \sin \left(p_{n} \omega\left[x_{n+1}-t\right]-p_{n+1} \omega\left[x-x_{n+1}\right]\right) / \omega .
\end{aligned}
$$

In these two cases we get respectively the estimates

$$
|\Phi(x, t, \lambda)| \leq \exp \left(|\operatorname{Im}(\omega)| p_{n}[x-t]\right)
$$

and

$$
\begin{aligned}
|\Phi(x, t, \lambda)| & \leq\left[1 / p_{n}+1 / p_{n+1}\right] \exp \left(|\operatorname{Im}(\omega)|\left(p_{n}\left[x_{n+1}-t\right]+p_{n+1}\left[x-x_{n+1}\right]\right)\right) \\
& \leq\left[1 / p_{n}+1 / p_{n+1}\right] \exp \left(|\operatorname{Im}(\omega)|\left(\left[p_{n}+p_{n+1}\right][x-t]\right)\right) .
\end{aligned}
$$

Using the representation (2.c) it is then straightforward to establish

Lemma 3.2. There are constants $K, P$ such that for all $0 \leq t \leq x \leq 1$ we have

$$
\begin{gathered}
|\Phi(x, t, \lambda)| \leq K \exp (|\operatorname{Im}(\omega)| P[x-t]), \\
|(d / d x) \Phi(x, t, \lambda)| \leq K \exp (|\operatorname{Im}(\omega)| P[x-t]),
\end{gathered}
$$

and for $|\lambda| \geq 1$

$$
|\Phi(x, t, \lambda)| \leq K \exp (|\operatorname{Im}(\omega)| P[x-t]) /|\omega| .
$$

Similarly we have the estimates

Lemma 3.3. There are constants $K, P$ such that for all $0 \leq x \leq 1$

$$
\begin{aligned}
& \left|y_{1}(x, \lambda)\right| \leq K \exp (|\operatorname{Im}(\omega)| P x), \\
& \left|y_{2}(x, \lambda)\right| \leq K \exp (|\operatorname{Im}(\omega)| P x), \\
& \left|y_{2}^{\prime}(x, \lambda)\right| \leq \exp (|\operatorname{Im}(\omega)| P x),
\end{aligned}
$$

and for $|\lambda| \geq 1$

$$
\begin{aligned}
& \left|y_{1}^{\prime}(x, \lambda)\right| \leq K|\omega| \exp (|\operatorname{Im}(\omega)| P x) \\
& \left|y_{2}(x, \lambda)\right| \leq K \exp (|\operatorname{Im}(\omega)| P x) /|\omega| .
\end{aligned}
$$

Based on these estimates, standard Picard iteration arguments [14, p. 331] can be used to derive estimates for $\varphi_{j}(x, \lambda)$ if $q(x) \in L^{1}[0,1]$. For instance if $\psi_{n}(x, \lambda)$ is defined by

$$
\begin{gathered}
\psi_{0}(x, \lambda)=y_{1}(x, \lambda), \\
\psi_{n}(x, \lambda)=y_{1}(x, \lambda)+\int_{0}^{x} \Phi(x, t, \lambda) p^{2}(t) q(t) \psi_{n-1}(t, \lambda) d t,
\end{gathered}
$$

one readily proves by induction that there are constants $K$ and $P$ such that

$$
\left|\psi_{n}-\psi_{n-1}\right|(x, \lambda) \leq \exp (|\operatorname{Im}(\omega)| P) K^{n+1}\left[\int_{0}^{x}|q(s)| d s\right]^{n} / n !
$$

Such arguments provide 
Lemma 3.4. Suppose that $q(x) \in L^{1}[0,1]$. The functions $\varphi_{j}^{(i-1)}(x, \lambda), i, j=$ 1,2 , are entire functions of $\lambda$ for each $x \in[0,1]$, with order $\frac{1}{2}$. There are constants $K, P$ such that for $|\lambda| \geq 1$

$$
\begin{aligned}
& \left|\varphi_{1}(x, \lambda)-y_{1}(x, \lambda)\right| \leq K \exp (|\operatorname{Im}(\omega)| P x) /|\omega|, \\
& \left|\varphi_{1}^{\prime}(x, \lambda)-y_{1}^{\prime}(x, \lambda)\right| \leq K \exp (|\operatorname{Im}(\omega)| P x), \\
& \left|\varphi_{2}(x, \lambda)-y_{2}(x, \lambda)\right| \leq K \exp (|\operatorname{Im}(\omega)| P x) /|\lambda|, \\
& \left|\varphi_{2}^{\prime}(x, \lambda)-y_{2}^{\prime}(x, \lambda)\right| \leq K \exp (|\operatorname{Im}(\omega)| P x) /|\omega| .
\end{aligned}
$$

Now we have all the ingredients needed for the proof of Theorem 1.1. Lemmas $3.1,3.3$, and 3.4 imply that

$$
\begin{aligned}
F_{q}(\lambda) & =d \varphi(1, \lambda)-c \varphi^{\prime}(1, \lambda) \\
& =-a c \varphi_{1}^{\prime}(1, \lambda)+\left[d a \varphi_{1}(1, \lambda)-b c \varphi_{2}^{\prime}(1, \lambda)\right]+b d \varphi_{2}(1, \lambda)
\end{aligned}
$$

is an entire function of order $\frac{1}{2}$, which is determined up to a nonzero scalar by the eigenvalues of problem (1.a). Suppose that $a c \neq 0$. By Lemma 3.4 if $\nu>0$ then

$$
\begin{aligned}
\lim _{L \rightarrow \infty} & {[2 / L] \int_{1}^{L} F_{q}(\lambda) \omega^{-1} \sin (\nu \omega) d \omega } \\
& =\lim _{L \rightarrow \infty}[2 / L] \int_{1}^{L}-\operatorname{ac} \varphi_{1}^{\prime}(1, \lambda) \omega^{-1} \sin (\nu \omega) d \omega \\
& =\lim _{L \rightarrow \infty}[2 / L] \int_{1}^{L}-a c y_{1}^{\prime}(1, \lambda) \omega^{-1} \sin (\nu \omega) d \omega \\
& =\lim _{L \rightarrow \infty}[2 / L] \int_{1}^{L} F_{0}(\lambda) \omega^{-1} \sin (\nu \omega) d \omega .
\end{aligned}
$$

In short, the presence of $q(x)$ has no affect on these limits and the proof follows from the previously established case when $q(x)=0$. If $a c=0$, the needed data is computed, as appropriate, from

$$
\lim _{L \rightarrow \infty}[2 / L] \int_{1}^{L} F_{q}(\lambda) \cos (\nu \omega) d \omega=\lim _{L \rightarrow \infty}[2 / L] \int_{1}^{L} F_{0}(\lambda) \cos (\nu \omega) d \omega
$$

or from

$$
\lim _{L \rightarrow \infty}[2 / L] \int_{1}^{L} F_{q}(\lambda) \omega \sin (\nu \omega) d \omega=\lim _{L \rightarrow \infty}[2 / L] \int_{1}^{L} F_{0}(\lambda) \omega \sin (\nu \omega) d \omega .
$$

\section{REFERENCES}

1. L. Andersson, Inverse eigenvalue problems with discontinuous coefficients, Inverse Problems 4 (1988), 353-397.

2. G. Birkhoff and G. Rota, Ordinary differential equations, Blaisdell, Waltham, MA, 1969.

3. G. Borg, Eine Umkehrung der Sturm-Liouville Eigenwertaufgabe, Acta Math. 76 (1946), $1-96$.

4. K. Chadan and P. C. Sabatier, Inverse problems in quantum scattering theory, SpringerVerlag, New York, 1989.

5. C. F. Coleman and J. R. McLaughlin, Solution of the inverse spectral problem for an impedance with integrable derivative, I, II, Comm. Pure Appl. Math. 46 (1993), 145-184, $185-212$. 
6. I. M. Gelfand and B. M. Levitan, On the determination of a differential equation by its spectral function, Amer. Math. Soc. Transl. Ser. 2, vol. 1, Amer. Math. Soc., Providence, RI, 1955, pp. 253-304.

7. H. Gottlieb, Iso-spectral operators: some model examples with discontinuous coefficients, J. Math. Anal. Appl. 132 (1988), 123-137.

8. O. Hald, Inverse eigenvalue problems, Comm. Pure Appl. Math. 37 (1984), 539-577.

9. I. S. Kac and M. G. Krein, On the spectral functions of the string, Amer. Math. Soc. Transl. Ser. 2, vol. 103, Amer. Math. Soc., Providence, RI, 1974, pp. 19-102.

10. R. Krueger, Inverse problems for nonabsorbing media with discontinuous material properties, J. Math. Phys. 23 (1982), 396-404.

11. E. R. Lapwood and T. Usami, Free oscillations of the earth, Cambridge Univ. Press, Cambridge, 1981.

12. J. R. McLaughlin, Analytical methods for recovering coefficients in differential equations from spectral data, SIAM Rev. 28 (1986), 53-72.

13. A. McNabb, R. Anderssen, and E. Lapwood, Asymptotic behaviour of the eigenvalues of a Sturm-Liouville system with discontinuous coefficients, J. Math. Anal. Appl. 54 (1976), 741-751.

14. R. Newton, Scattering theory of waves and particles, McGraw-Hill, New York, 1966.

15. J. Poeschel and E. Trubowitz, Inverse spectral theory, Academic Press, Orlando, 1987.

16. R. Young, An introduction to nonharmonic Fourier series, Academic Press, New York, 1980.

Department of Mathematics, University of Colorado at Colorado Springs, ColORADO SPRINGS, COLORADO 80933

E-mail address: Carlson@vision.uccs.edu 